\title{
Multifocal Post-Traumatic Myositis Ossificans Circumscripta in a Young Male Following a Motor Vehicle Accident: A Review of Imaging and Clinical Presentation
}

\author{
Ahjay Bhatia ${ }^{1}$, Ashley Ryan Vidad ${ }^{2}$, Divy Mehra ${ }^{3}$, Himadri Shah ${ }^{4}$, Oluwaseun Ogunjemilusi ${ }^{5}$ \\ 1. Osteopathic Medicine, Nova Southeastern University Dr. Kiran C. Patel College of Osteopathic Medicine, Plantation, \\ USA 2. Family Medicine, Internal Medicine, Surgery, Pathology, Gynecology, Infectious Disease, Neurology, \\ Orthopedics, Gastroenterology, Nova Southeastern University Dr. Kiran C. Patel College of Osteopathic Medicine, Fort \\ Lauderdale, USA 3. Ophthalmology, Nova Southeastern University Dr. Kiran C. Patel College of Osteopathic Medicine, \\ Fort Lauderdale, USA 4. College of Osteopathic Medicine, Nova Southeastern University Dr. Kiran C. Patel College of \\ Osteopathic Medicine, Davie, USA 5. Internal Medicine, Westside Regional Medical Center, Plantation, USA
}

Corresponding author: Ahjay Bhatia, ahjaybhatia@yahoo.com

\begin{abstract}
Traumatic myositis ossificans (MO) circumscripta is an uncommon nonhereditary pathophysiological result of muscular trauma that is detected by radiographic imaging three to four weeks following initial trauma. It is responsible for great global morbidity, with symptoms of prolonged pain, diminished flexibility, and stiffness. There is frequently a delay in diagnosis due to the generalized symptoms and varying radiographic presentation. The goal of therapy is to rule out serious complications (such as soft tissue sarcoma) and to restore strength and range of motion (ROM) as soon as possible. Here we detail the case of a 32-year-old male with a delayed diagnosis of MO who presented to the hospital with left lower extremity pain and swelling following a motor vehicle accident (MVA) that occurred one month prior.
\end{abstract}

Review began 03/15/2021 Review ended 03/29/2021 Published 04/06/2021

\section{(c) Copyright 2021}

Bhatia et al. This is an open access article distributed under the terms of the Creative Commons Attribution License CC-BY 4.0., which permits unrestricted use, distribution, and reproduction in any medium, provided the original author and source are credited.
Categories: Pathology, Radiology, Orthopedics

Keywords: myositis ossification, post trauma, radiological findings, radiological diagnosis, bone pathology, radiology, non-surgical orthopedics, trauma imaging, anatomic imaging, myositis ossificans

\section{Introduction}

Traumatic myositis ossificans (MO) circumscripta is an inflammatory disorder that arises in skeletal muscle [1]. It is a benign, solitary, self-limiting disorder representing metaplasia of bone. Traumatic MO circumscripta represents one of three distinct disorders that are all characterized by the development of ectopic bony tumors in different skeletal muscle locations: MO progressive, traumatic MO circumscripta, and MO circumscripta without history of trauma [2]. The pathophysiology of MO circumscripta formation is not completely understood but is thought to occur through differentiation of fibroblasts into osteogenic cells [2]. Kan et al. [3] demonstrated that the cellular mechanism of heterotopic bone formation is the result of the dysregulation of local stem cells in response to tissue injury and inflammation. Men and women are equally affected with no preferential anatomical site, though clinical cases in the girdles and limbs are frequently described [1].

The diagnosis of MO is often difficult to make and regularly overlooked. Early MO without a considerable amount of calcification and ossification can lead to either false negative radiological readings or misdiagnosis as other, more serious conditions (osteosarcoma) [4]. A high level of clinical suspicion is necessary as complaints are nonspecific (e.g. pain, swelling) and diagnosis is predominantly radiological. Treatment is conservative, as the process tends to be self-limiting and self-resolving, and generally consists of nonsteroidal anti-inflammatory drugs (NSAIDs) and physical therapy [5-6]. Here, we detail the delayed diagnosis of traumatic MO circumscripta in a young male following a motor vehicle accident (MVA) to further highlight and raise clinical suspicion for the disease in post-traumatic patients.

\section{Case Presentation}

A 32-year-old male with no chronic medical conditions presented to the ED complaining of worsening left lower extremity swelling and pain. One month prior to presentation, this gentleman suffered an MVA, presented to the ED, and was discharged after exclusion of a deep vein thrombosis (DVT).

During the current visit, the patient was afebrile, and his vitals were stable. On physical exam, the left lower extremity was edematous and warm to touch with normal pulses, no limitations in range of motion (ROM), no calf tenderness, no motor or sensory deficits, and no other neurologic abnormalities. A venous duplex ultrasound of both lower extremities was ordered and showed no sonographic evidence of DVT. Laboratory workup revealed abnormal renal function (estimated glomerular filtration rate, GFR of 39, blood urea 


\section{Cureus}

nitrogen, BUN of $37 \mathrm{mg} / \mathrm{dL}$, creatinine of $2.00 \mathrm{mg} / \mathrm{dL}$ ), transaminitis [aspartate aminotransferase (AST) of $107 \mathrm{U} / \mathrm{L}$ ], an elevated D dimer, mildly elevated parathyroid hormone (PTH), and mild decreased values of red blood cell (RBC) count (3.36*106/uL), hemoglobin (10.1 g/dL), and hematocrit (30.4\%). A bilateral renal artery ultrasound was performed to check for signs of hydronephrosis but it was negative and showed increased echogenicity of the renal cortices, correlating clinically for chronic medical renal disease. Based on the clinical picture and laboratory findings, we made a diagnosis of mild acute kidney injury and rhabdomyolysis with secondary hyperparathyroidism and transaminitis. The subsequent plan was to administer pain control with acetaminophen $500 \mathrm{mg}$ as needed, cholecalciferol (vitamin D3) $25 \mathrm{mcg}$ daily, IV normal saline at $100 \mathrm{cc} / \mathrm{h}$, and prophylactic antibiotics (azithromycin and ceftriaxone), as well as to consult nephrology.

Despite this hospital management, the patient's leg pain and swelling progressively worsened and after three days a CT scan with contrast of the left lower extremity was ordered. The CT revealed subcutaneous and intrafascial edema (particularly in the anterior compartment of the proximal thigh); further, curvilinear calcifications within the distal vastus intermedius, vastus lateralis, and vastus medialis (Figure 1), proximal lateral gastrocnemius, soleus, fibularis longus (Figure 2), flexor hallucis longus, and tibialis posterior muscles. A cleft was noted between these calcifications and underlying bone. The overall impression was of MO involving the distal anterior compartment of the thigh and posterior anterior compartment of the lower leg, suspected as a result of prior trauma (MVA). An orthopedic consultation was organized for follow up in the outpatient setting and the patient was subsequently discharged after being administered lidocaine patches and discussing the avoidance of NSAIDs and aspirin until the resolution of renal issues.

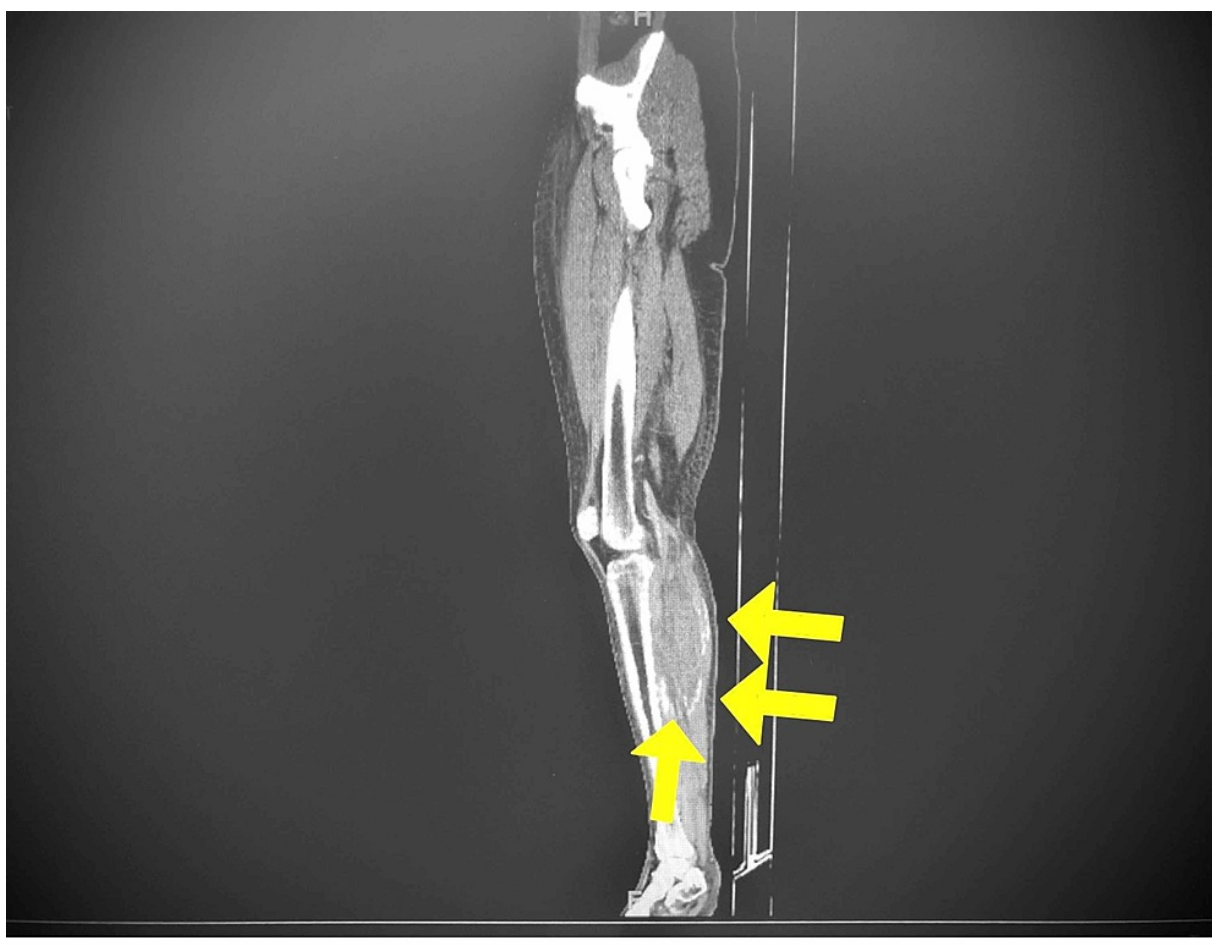

FIGURE 1: CT (sagittal view) of the left lower extremity.

Curvilinear calcifications within the proximal lateral gastrocnemius and soleus muscles. 


\section{Cureus}

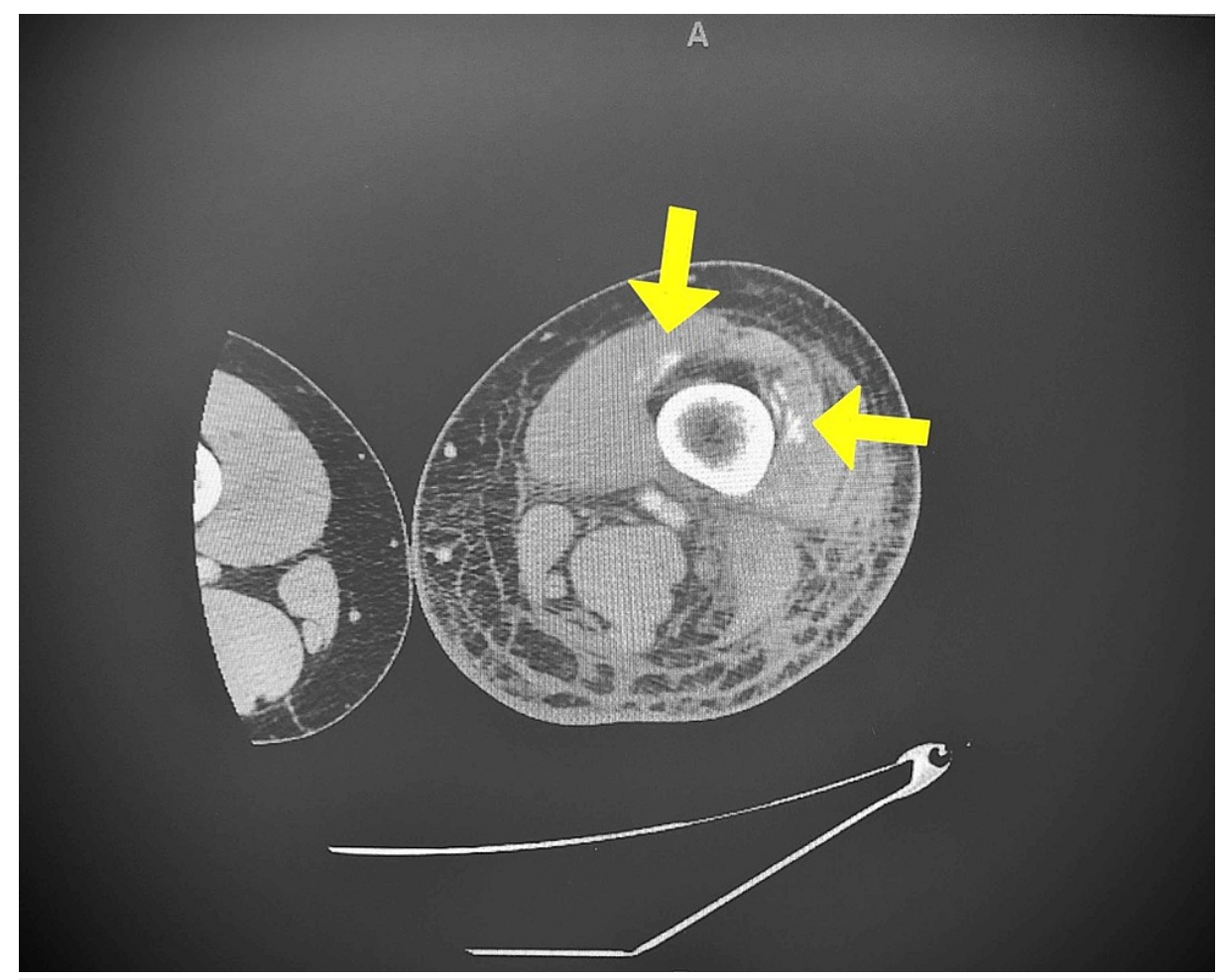

FIGURE 2: CT (axial view) of the left lower extremity.

Curvilinear calcifications within the distal vastus intermedius, vastus lateralis, and vastus medialis.

\section{Discussion}

Traumatic MO circumscripta is a benign, self-limiting condition characterized by heterotopic bone formation, usually involving striated muscle [5]. There is equal occurrence in both men and women with adolescents and young adults under 30 being the most commonly affected [7]. The diagnosis of MO is made clinically and radiologically.

Both the clinical presentation and radiological findings of MO vary and are correlated with the stage of disease progression of the patient [4]. The early stage (<4 weeks) is characterized by diffuse pain and swelling and there is little to no osteoid formation [2]. These generalized symptoms make it difficult for clinicians to pick up MO early in the disease course, as well as for radiologists to identify the disease on imaging. For these reasons, most cases of MO are typically diagnosed three to four weeks after the initial trauma with MRI being the preferred modality [5]. The intermediate stage of MO (four to eight weeks) is characterized by immature osteoid formation, which gradually changes into mature bone on the periphery of the mass, and the mature stage (> 8 weeks) includes mature lamellar bone [8].

Radiographic findings of MO are important to recognize as they can often be confused with or misdiagnosed as more sinister conditions like osteosarcoma [9]. Peripheral, curvilinear calcifications (Figure 1) allow for distinction between the two pathologies, as central calcifications are more characteristic for bone forming sarcomas [9]. Additionally, the extensive muscle edema characteristic of the early stage of MO is unusual in most primary neoplasms [9]. Intralesional post-contrast enhancement, however, may lead the clinician to favor osteosarcoma, although such descriptions have been noted in past MO cases [10]. A misdiagnosis as a neoplasm can lead to unnecessary biopsy of the ossification and can worsen prognosis of MO [10-11].

Treatment of MO is supportive as the disease is self-limiting. NSAIDs and physical therapy are the mainstays of treatment to reduce swelling and increase ROM of the affected area, especially in cases involving the extremities [12]. Surgical intervention is only necessary when the patient has unbearable pain or when secondary complications (nerve impingement) arise [12]. In the current case, NSAIDs were avoided to a history of renal issues. Alternative analgesics like acetaminophen or opiates can be used, but NSAIDs are preferred when indicated due to their anti-inflammatory properties.

\section{Conclusions}

Traumatic MO circumscripta can go undiagnosed or be misdiagnosed due to nonspecific symptoms on clinical presentation, as well as due to the inconsistent findings on imaging (a result of the progression of 
the disease). As such, it is important to have a high level of clinical suspicion in post-traumatic patients to catch the disease early in its disease course.

\section{Additional Information \\ Disclosures}

Human subjects: Consent was obtained or waived by all participants in this study. Conflicts of interest: In compliance with the ICMJE uniform disclosure form, all authors declare the following: Payment/services info: All authors have declared that no financial support was received from any organization for the submitted work. Financial relationships: All authors have declared that they have no financial relationships at present or within the previous three years with any organizations that might have an interest in the submitted work. Other relationships: All authors have declared that there are no other relationships or activities that could appear to have influenced the submitted work.

\section{References}

1. Landolsi M, Mrad T: Traumatic myositis ossificans circumscripta (MOC). BMJ Case Rep. 2017, 2017:bcr2017-219422. 10.1136/bcr-2017-219422

2. Walczak BE, Johnson CN, Howe BM: Myositis ossificans. J Am Acad Orthop Surg. 2015, 23:612-622. 10.5435/JAAOS-D-14-00269

3. Kan L, Liu Y, McGuire TL, Berger DM, Awatramani RB, Dymecki SM, Kessler JA: Dysregulation of local stem/progenitor cells as a common cellular mechanism for heterotopic ossification. Stem Cells. 2009, 27:150-156. 10.1634/stemcells.2008-0576

4. Wang H, Nie P, Li Y, Hou F, Dong C, Huang Y, Hao D: MRI findings of early myositis ossificans without calcification or ossification. Biomed Res Int. 2018, 2018:4186324. 10.1155/2018/4186324

5. Lipscomb AB, Thomas ED, Johnston RK: Treatment of myositis ossificans traumatica in athletes . Am J Sports Med. 1976, 4:111-120.

6. Torrance DA, Degraauw C: Treatment of post-traumatic myositis ossificans of the anterior thigh with extracorporeal shock wave therapy. J Can Chiropr Assoc. 2011, 55:240-246.

7. El Bardouni A, Boufettal M, Zouaidia F, Kharmaz M, Berrada MS, Mahassini N, El Yaacoubi M: Nontraumatic myositis ossificans circumscripta: a diagnosis trap. J Clin Orthop Trauma. 2014, 5:261-265 10.1016/j.jcot.2014.09.005

8. Lee KR, Park SY, Jin W, Won KY: MR imaging and ultrasonography findings of early myositis ossificans: a case report. Skeletal Radiol. 2016, 45:1413-1417. 10.1007/s00256-016-2441-4

9. Luczynska E, Kasperkiewicz H, Domalike A: Myositis ossificans mimicking sarcoma, the importance of diagnostic imaging-case report. Pol J Radiol. 2014, 79:228-232.

10. Kransdorf MJ, Meis JM: From the archives of the AFIP. Extraskeletal osseous and cartilaginous tumors of the extremities. Radiographics. 1993, 13:853-884. 10.1148/radiographics.13.4.8356273

11. Goyal K, Pettis CR, Bancroft AE, Wasyliw CW, Scherer KF: Myositis ossificans in the thigh of a lacrosse player. Orthopedics. 2015, 38:468-518. 10.3928/01477447-20150804-01

12. Kransdorf MJ, Murphey MD: Radiologic evaluation of soft-tissue masses: a current perspective . Am J Roentgenol. 2000, 175:575-587. 10.2214/ajr.175.3.1750575 